\title{
Assessment of 3-D Printing Technologies for Millimeter-Wave Reflectors
}

\author{
Jordi Romeu ${ }^{\circledR}$, Fellow, IEEE, Sebastián Blanch ${ }^{\circledR}$, Neus Vidal ${ }^{\circledR}$, \\ Josep Maria Lopez-Villegas, Senior Member, IEEE, and Albert Aguasca, Member, IEEE
}

\begin{abstract}
Three different 3-D printing technologiesstereolithography, fused deposition modeling, and HP Multi Jet Fusion technology-are compared to build a parabolic reflector operating at $100 \mathrm{GHz}$. Fabrication tolerance and surface roughness before and after metallization are accurately measured. The performance of the reflectors is measured in the near field, and it is compared against an optical grade reflector. In this way, the performance of the final product is thoroughly assessed.
\end{abstract}

Index Terms-Millimeter-wave devices, reflector antennas, three-dimensional printing.

\section{INTRODUCTION}

A DDITIVE manufacturing technologies have become an effective alternative for the manufacturing of antennas. The major challenge in producing antennas in millimeter- and submillimeter-wave regions is to ensure the accuracy in the manufacturing [1], [2]. In addition, in the particular case of reflector manufacturing, the metallization process has to be also taken into account. Surface reflector roughness is a major source of gain reduction in a reflector. The well-known Ruze's formula [3] expresses the gain loss or reflector surface efficiency as

$$
\Delta G=-685.81\left(\frac{\epsilon}{\lambda}\right)^{2}(d B)
$$

where $\varepsilon$ is the root mean square (rms) surface error and $\lambda$ is the wavelength. It means that at a frequency of $100 \mathrm{GHz}$, the rms error has to be smaller than $36 \mu \mathrm{m}$ to have a gain loss smaller than $0.1 \mathrm{~dB}$. Three-dimensional (3-D) printers have resolutions of the order of 10-100 $\mu \mathrm{m}$; therefore, it is interesting to measure the accuracy of different printing technologies to determine the upper frequency limit in which they can be used to print reflectors. To this end, three different printing technologiesstereolithography (SLA), fused deposition modeling (FDM), and HP Multi Jet Fusion (MJF) technology_are compared.

Manuscript received April 12, 2018; revised May 22, 2018 and June 29, 2018; accepted July 17, 2018. Date of publication; date of current version. This work was supported in part by the Spanish Inter-Ministerial Commission on Science and Technology (CICYT) under Grant TEC201678028-C3-1-P, Grant TIN2014-55413-C2-1-P, Grant TEC2017-85244-C2-2-P, and Grant TEC201783524-R, and in part by FEDER and the Unidad de Excelencia Maria de Maeztu under Grant MDM-2016-0600, which is financed by the Agencia Estatal de Investigación, Spain. (Corresponding author: Jordi Romeu.)

J. Romeu, S. Blanch, and A. Aguasca are with the Department of Signal Theory and Communications, CommSensLab, Universitat Politècnica de Catalunya (UPC), Barcelona 08034, Spain (e-mail: romeu@tsc.upc.edu; blanch@tsc.upc. edu; aguasca@tsc.upc.edu).

N. Vidal and J. M. Lopez-Villegas are with the Department of Electronic and Biomedical Engineering, University of Barcelona, Barcelona 08007, Spain (e-mail: nvidal@ub.edu; j.m.lopez-villegas@ub.edu).

Digital Object Identifier 10.1109/LAWP.2018.2857856

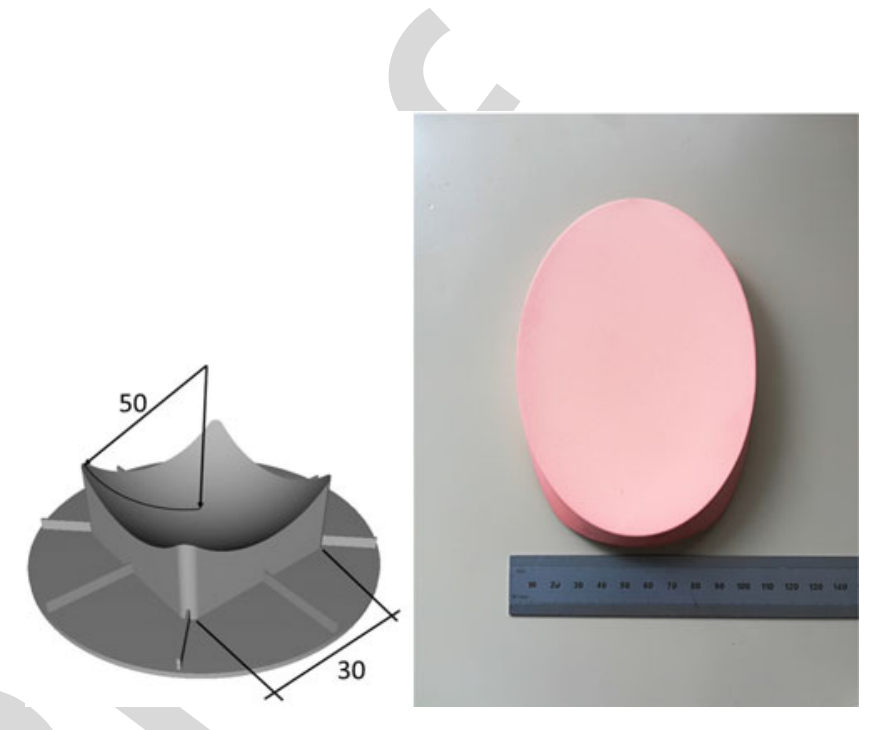

Fig. 1. Test object (left) and the metallized reflector (right).

A $90^{\circ}$ offset parabolic reflector has been printed and metallized. The geometry of the reflector has been chosen to be the same as a commercial optical grade reflector, so their performance can be benchmarked against it. The mechanical accuracy of the printed and metallized surfaces has been measured by a confocal optical profiler that is able to provide accurate contactless surface profiles. Finally, a planar near-field scan of the reflectors has been done to assess their electromagnetic performance. The paper is organized as follows. In Section II, the 3-D printing, metallization, and mechanical verification of the printed reflectors are described. In Section III, the electromagnetic behavior of the reflectors is presented, and finally, the conclusions are presented in Section IV.

\section{MANUFACTURING PROCESS}

\section{A. 3-D Printing and Metallization}

Two sets of objects have been printed and metallized. The first is the test object shown in Fig. 1 (left). It is a sphere of radius $50 \mathrm{~mm}$ intersected with a cube of $30 \mathrm{~mm}$ side. This test object has the advantage that its measured profile can be easily compared with the theoretical one.

The second object shown in Fig. 1 (right) is a $90^{\circ}$ offset reflector of $101.6 \mathrm{~mm}$ diameter with a parent focal length of $76.2 \mathrm{~mm}$. This geometry has been chosen to be the same as commercial optical grade reflector made by Edmund Optics that will be used as a benchmark for the 3-D-printed reflectors. The reference parabolic reflector has nominal rms roughness smaller than $0.01 \mu \mathrm{m}$ and has an Aluminum 6061-T6 coating with a 
TABLE I

SURFACE ERROR FOR THE TEST OBJECT

\begin{tabular}{llllr}
\hline \hline Name & $\begin{array}{c}\text { Printing } \\
\text { Technology }\end{array}$ & Material & Finishing & $\begin{array}{c}\text { Surface } \\
\text { error } \\
\text { rms } \\
(\mu \mathrm{m})\end{array}$ \\
\hline FDM & FDM & PLA & None & 27.6 \\
FDM+Cu & FDM & PLA & Sanding, & 13.8 \\
SLA+Cu & SLA & Therma & Metallization & \\
MJF1 & MJF & PA 12 & Sand Blasting & 8.8 \\
MJF2 & MJF & PA 12 & Tumbling & 24.5 \\
MJF3 & MJF & PA 12 GB & Sand Blasting & 12.2 \\
MJF4 & MJF & PA 12 GB & Tumbling & 36 \\
\hline \hline
\end{tabular}

conductivity of $2.510^{7} \mathrm{~S} / \mathrm{m}$. The SLA objects have been printed using a XFAB Stereolithographic 3-D printer from DWS. The base material is a nanoceramic-filled photopolymer Therma 294 that allows high resolution modeling (10-100 $\mu \mathrm{m}$ layer thickness). For the FDM, a SIGMA 3-D printer manufactured by BCN3D with a step resolution of $100 \mu \mathrm{m}$ has been used and the objects have been printed on polylactide (PLA). Finally, an HP 3-D MJF 4200 has been used to print the objects on two different materials PA 12 and PA 12 GB. These are thermoplastics the second one with a loading of glass beads to increase the mechanical stability. The MJF printed objects have been given two different finishing processes, sandblasting and tumbling, to reduce the surface roughness. The metallization process is by copper electrodeposition by electrolysis. A $17 \mu \mathrm{m}$ thick layer of copper is deposited following the process described in [4]. Further testing has shown that this method provides surface resistances close to the ones obtained from copper, in particular accurate cavity measurements at $9 \mathrm{GHz}$ have shown a surface resistance of $35.93 \mathrm{~m} \Omega$ for electrodeposited copper on PLA compared to $25.68 \mathrm{~m} \Omega$ for pure copper [5].

\section{B. Mechanical Verification}

The accuracy of the printed objects has been verified by a Plu Neox Optical Profiler manufactured by Sensofar Metrology [6]. The measurement principle is described in [7] and it allows contactless high accuracy profile measurements that include submicron surface roughness measurements. The goal of the mechanical verification is to have a measurement of the surface roughness as well as deviations from the specified nominal shape. To this end, accurate profiles of the test object of Fig. 1 (left) have been measured before and after metallization. The description of each object is shown in Table I and the measured results are shown in Fig. 2. For each one of the test objects, the measured profile compared to the theoretical one is shown on the left of the figure. On the right, the surface error is shown. From this error curve, the rms surface error is found and it is shown in Table I. The results show that the best roughness is obtained by the SLA printed object that has a roughness of $8 \mu \mathrm{m}$ after metallization. The profile measurements of Fig. 2 also show the effects on the surface of the applied surface treatment. In the case of the MJF samples, sandblasting or tumbling has been applied. It is observed that these surface treatments smoothen the surface, but they can leave residual surface errors. In the
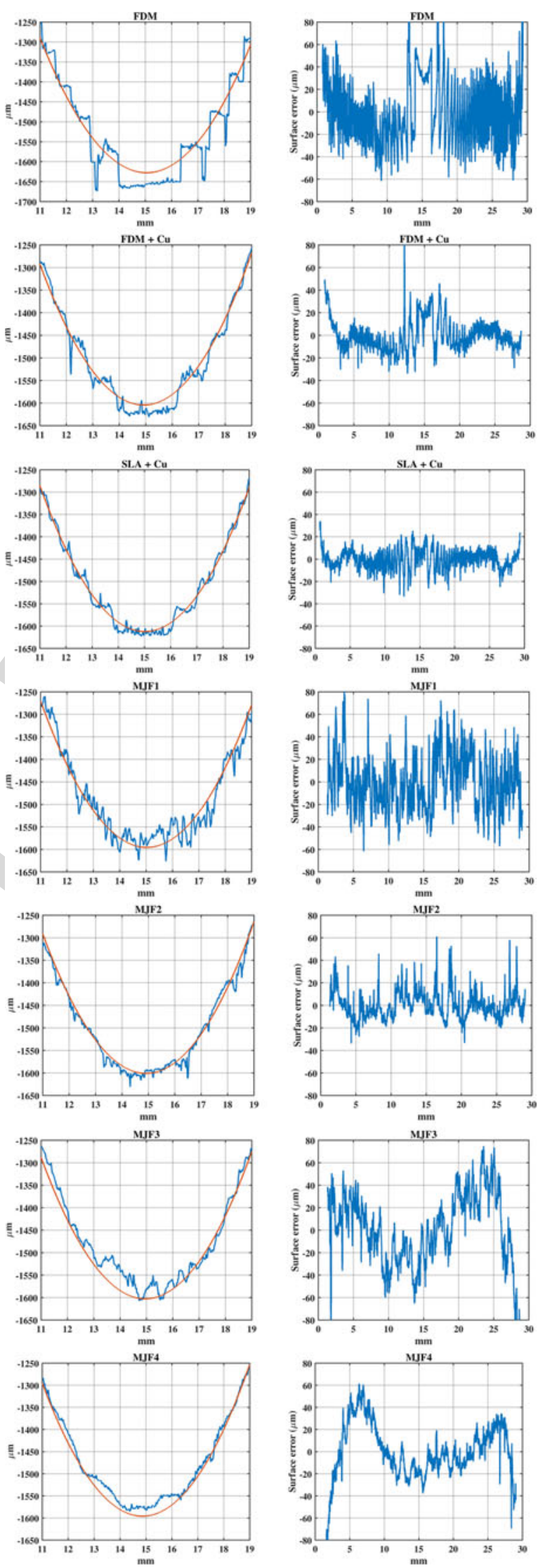

Fig. 2. Profile measurement results for the test object (left) and surface error (right). 


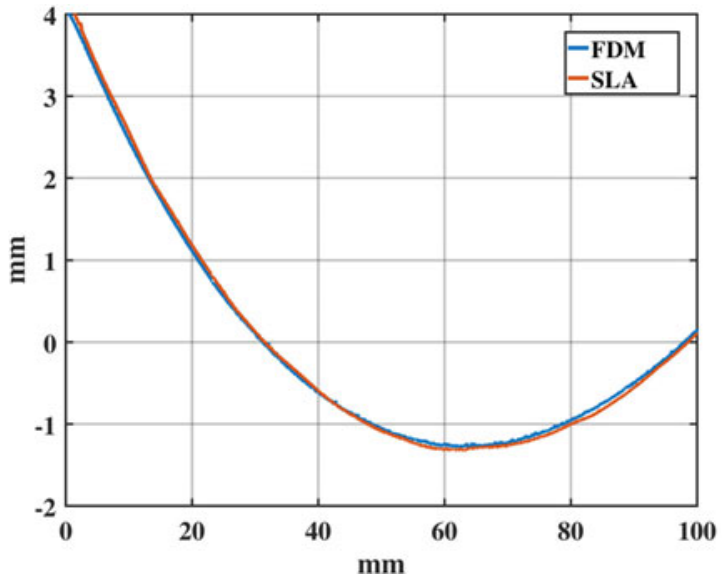

Fig. 3. Profile measurement of the metallized FDM and SLA printed reflectors.

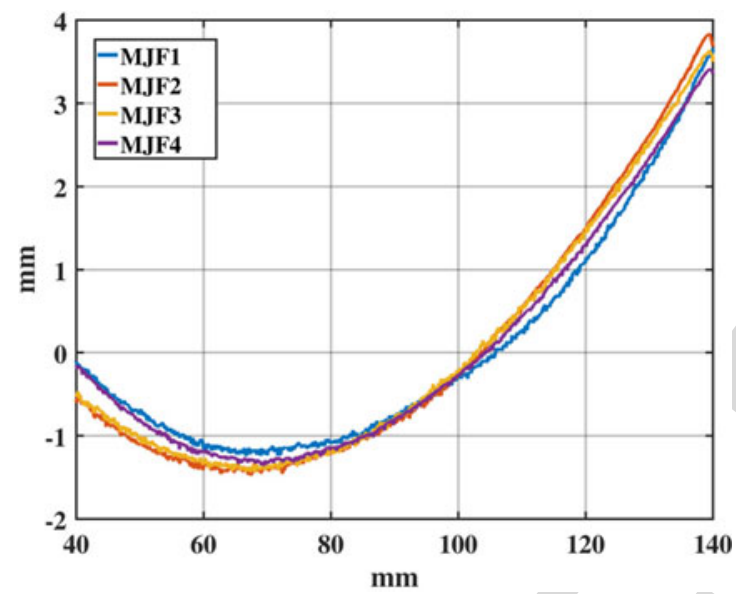

Fig. 4. Profile measurement of the metallized MJF printed reflectors.

case of the FDM object, the $100 \mu$ m vertical steps of the printer are clearly observable. For this object, prior to the metallization, it has been smoothened with a fine grain sandpaper. Therefore, the resulting metallized object has a smoother texture. In all cases, the surface roughness is below the $36 \mu \mathrm{m}$ design goal for a $100 \mathrm{GHz}$ reflector.

Once the reflectors have been printed and metallized, and before proceeding to the EM testing, their profile has also been measured with the optical profiler. Due to the large dimension of the reflector, a partial profile along the vertical dimension has been measured. In Fig. 3, the profiles for the FDM and SLA printed and metallized reflectors are compared. In Fig. 4, the profiles for the four MJF reflectors are compared. The first evident conclusion is that the four MJF reflectors present clear differences in their profiles. It is also evident that their roughness is higher than in the FDM and SLA printed reflectors. For unknown reasons, that has to be further investigated as some of the MJF reflectors have suffered some deformation during the printing process.

\section{EM TESTING}

In order to assess the performance of the reflectors, their radiation pattern has been measured using a planar near field scanning technique at a frequency of $100 \mathrm{GHz}$. The measurement setup

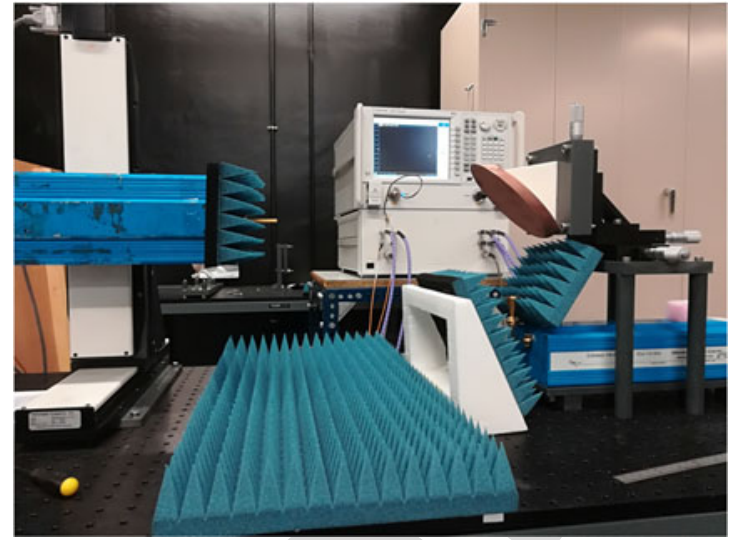

Fig. 5. Near-field scanning of a printed reflector.

is shown in Fig. 5 where one of the 3-D printed reflectors is tested.

The $H$ plane pattern (horizontal cut according to the measurement setup of Fig. 5) for all the reflectors is shown in Fig. 6. In Table II, the directivity and the normalized radiated power for each reflector compared to the reference optical grade reflector is shown. Notice that, in this case, all the printed reflectors have been metallized. The observation of the radiation patterns shows similar cross-polar level in all cases. The changes in directivity can be as high as $-0.80 \mathrm{~dB}$ compared to the optical grade reflector and the reflector that better matches the performance of the optical grade reflector is the FDM. The total radiated power has been compared from the integration of the field components in the near-field measurements. It is interesting to note that the radiated power for the 3-D printed reflectors is higher than the power radiated by the optical grade reflector in all cases.

This fact can be explained due to the lower surface resistance of the copper metallization compared to the aluminum coating of the optical reflector. In addition, the thickness of the aluminum coating of the optical grade reflector is not known, but it is also possible that the coating thickness is smaller than $30 \mu \mathrm{m}$, which is the penetration depth at $100 \mathrm{GHz}$, finally the fact that the optical grade reflector has sharper edges that probably contribute to higher edge diffraction. The radiated power is obtained from the planar near-field measurement; therefore, the scattered power is not properly taken into account. In Table II, the measured roughness for each reflector is also shown. This roughness has been measured following the procedure of [8]. The roughness is the rms height after removing the primary surface. The specific way in which it has been computed involves two steps. First an error function is obtained by subtracting the desired parabolic curve from the measured profile. Then the rms value of the error function is obtained after applying a spatial low pass filter of $2 \mathrm{~mm}$ cutoff length. It is observed that after metallization, the surface roughness is below $21 \mu \mathrm{m}$ in all cases. As expected from the results of Figs. 3 and 4, the roughness for the SLA and FDM reflectors is smaller. It is also observed that the $H$ plane $3 \mathrm{~dB}$ beam width (horizontal plane) is practically the same in all cases, and differences of the order of $0.1^{\circ}$ can be observed in the $E$ plane. Of course the larger beam width increments correspond to the largest decrements in directivity. Due to the similar surface roughness, we think that the directivity reduction is produced by larger scale surface errors. As shown 

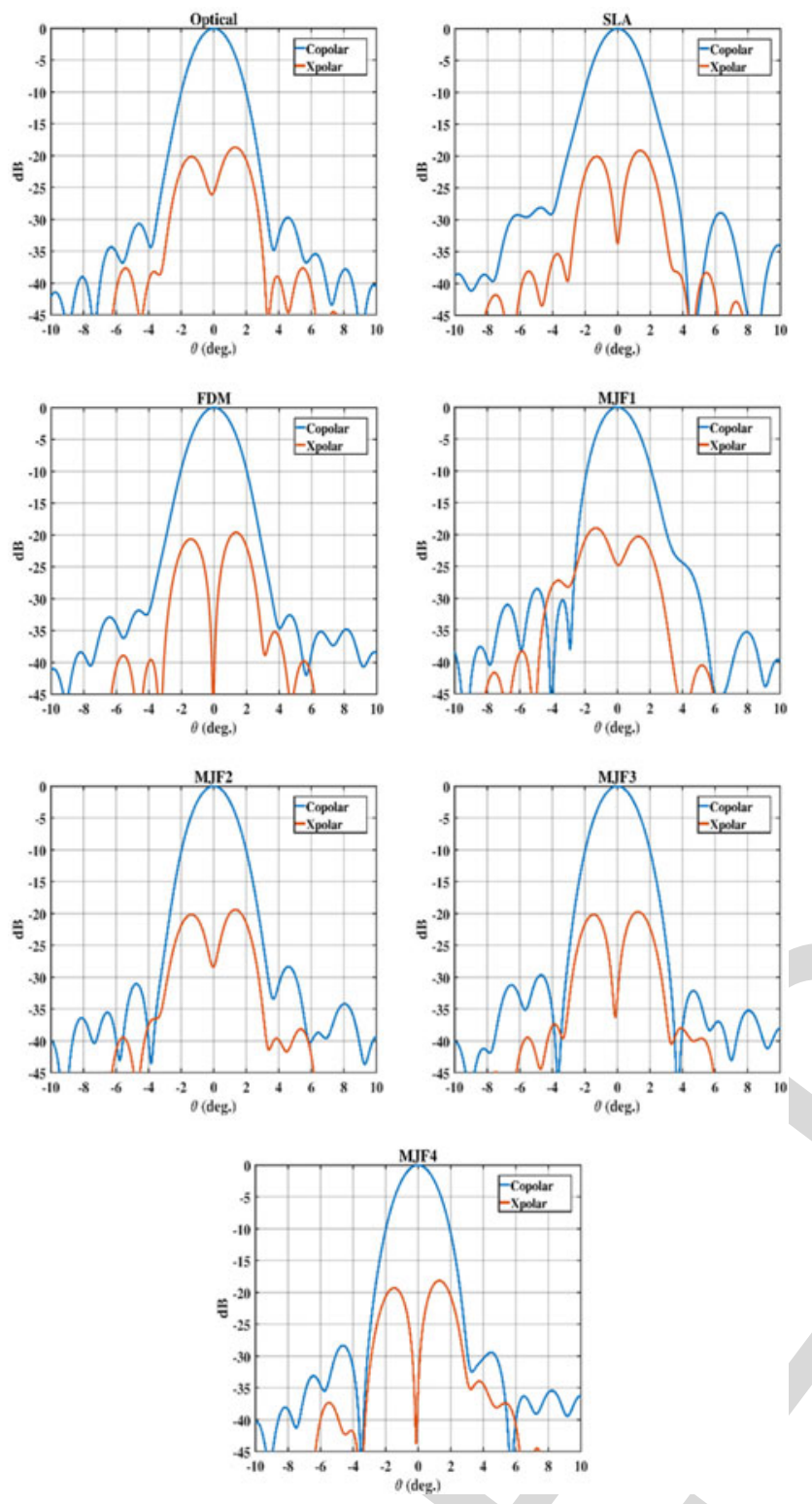

Fig. 6. $\quad H$ plane radiation pattern for each reflector. in Fig. 4, the profiles of the MJM-printed reflectors exhibit similar profiles and their directivity loss compared to the optical reflector is similar. On the other hand, MJM1 and MJM4 have more different profiles that we must infer that have higher deviation from the nominal surface that result in higher directivity losses.

Assuming that the FDM reflector is the one that better reproduces the nominal reflector shape, the comparison of Fig. 3 shows that the SLA profile has a ripple around the nominal shape. For practical reason, the SLA reflector was printed vertically and that resulted in this ripple that can be the cause of the directivity reduction. large differences. As a matter of fact, MJM2 and MJM3 have
TABLE II

MEASURED RADIATION PARAMETERS

\begin{tabular}{lcrrrrr}
\hline \hline Name & $\begin{array}{c}\mathrm{D} \\
(\mathrm{dB})\end{array}$ & $\begin{array}{c}\Delta \theta_{3 \mathrm{~dB}} \\
\text { E- } \\
\text { plane } \\
\left({ }^{\circ}\right)\end{array}$ & $\begin{array}{c}\Delta \theta_{3 \mathrm{~dB}} \\
\mathrm{H}-\text { plane } \\
\left({ }^{\circ}\right)\end{array}$ & $\begin{array}{c}\text { Relative } \\
\text { Directivity } \\
(\mathrm{dB})\end{array}$ & $\begin{array}{c}\text { Relative } \\
\text { Radiated } \\
\text { Power } \\
(\mathrm{dB})\end{array}$ & $\begin{array}{c}\text { Roughness } \\
(\mu \mathrm{m})\end{array}$ \\
\hline Optical & 38.84 & 2.02 & 2.22 & 0 & 0 & $0.01^{1}$ \\
FDM & 38.83 & 2.03 & 2.26 & -0.01 & 0.21 & 9.6 \\
SLA & 38.33 & 2.13 & 2.27 & -0.51 & 0.56 & 8 \\
MJF1 & 38.39 & 2.13 & 2.23 & -0.45 & 0.34 & 17 \\
MJF2 & 38.61 & 2.07 & 2.23 & -0.23 & 0.2 & 14 \\
MJF3 & 38.69 & 2.05 & 2.23 & -0.15 & 0.4 & 21 \\
MJF4 & 38.04 & 2.18 & 2.22 & -0.80 & 0.36 & 14 \\
\hline \hline
\end{tabular}

${ }^{1}$ Nominal value

\section{CONCLUSION}

The potentiality of 3-D printing of parabolic reflectors for 185 being used in frequencies in the $100 \mathrm{GHz}$ band has been shown. 186 Accurate surface measurements have shown that the metallized 187 reflectors can achieve surface roughness of the order of $10 \mu \mathrm{m} . \quad 188$ According to Ruze's equation, a reflector with such rough- 189 ness could be used for frequencies up to $300 \mathrm{GHz}$ with gain 190 losses of $0.1 \mathrm{~dB}$. Nevertheless, the measurements have shown 191 that although the local roughness can achieve these low values, 192 there may be other larger scale surface errors that can degrade 193 the performance of the reflector. In particular, the best results 194 have been obtained with the FDM reflector that has almost the 195 same performance as the optical grade reflector. In this case, 196 although the printing resolution is not the best, the fact that 197 the printing material is relatively soft leads to easy smooth- 198 ing by hand sanding. Also the printing material PLA does not 199 need high temperatures and that may explain the smaller de- 200 formation of the printed reflector as compared to the HP MJF 201 reflectors.

\section{REFERENCES}

[1] E. A. Rojas-Nastrucci, J. T. Nussbaum, N. B. Crane, and T. M. Weller, 204 "Ka-Band characterization of binder jetting for 3-D printing of metal- 205 lic rectangular waveguide circuits and antennas," IEEE Trans. Mi- 206 crow. Theory Techn., vol. 65, no. 9, pp. 3099-3108, Sep. 2017, doi: 207 10.1109/TMTT.2017.2730839.

[2] B. Zhang, Y. X. Guo, H. Zira on 3-D-printing technologies for millimeter-wave and terahertz appli- 210 cations," in Proc. IEEE, 2017, vol. 105, no. 4, pp. 723-736, doi: 211 10.1109/JPROC.2016.2639520.

[3] J. Ruze, "Antenna tolerance theory-A review," in Proc. IEEE, 1966, 213 vol. 54, no. 4, pp. 633-640, doi: 10.1109/PROC.1966.4784.

[4] J. Romeu, A. Aguasca, S. Blanch, J. O’Callaghan, L. Jofre, and S. Buitrago, "A submillimeter wave parabolic reflector by additive manufacturing," in Proc. Accepted IEEE Antennas Propag. Soc. Symp., Boston, 2018.

[5] P. Krkotic, A. Aguasca, and J. M. O'Callaghan, "Small footprint evaluation of metal coatings for additive manufacturing," in Proc. Submitted Eur. Microw. Conf., Madrid, 2018.

[6] Sensofar Group. [Online]. Available: www.sensofar.com

[7] R. Artigas, A. Pintó, and F. Laguarta, "Three-dimensional micromeasurements on smooth and rough surfaces with a new confocal optical profiler SPIE," in Proc. Int. Soc. Opt. Eng., 1999, vol. 3824, pp. 93-104.

[8] Geometrical Product Specifications (GPS)—Surface Texture: Areal-Part 225 2: Terms, Definitions and Surface Texture Parameters, ISO 25178- 226 2:2012(en).

\section{. .

\section{,} (1) , . , 01} 3 4 6 08 10 12 213 214 227 
- Authors: We cannot accept new source files as corrections for your paper. If possible, please annotate the PDF proof 229 we have sent you with your corrections and upload it via the Author Gateway. Alternatively, you may send us your 230 corrections in list format. You may also upload revised graphics via the Author Gateway.

Q1. Author: Please confirm or add details for any funding or financial support for the research of this article.

Q2. Author: The sense of the sentence "these are thermoplastics" is not clear. Please check that it reads correctly and supply a 234 revised version if necessary.

Q3. Author: Please provide the expansion of EM at first mention.

Q4. Author: The sense of the sentence "In addition, the thickness" is not clear. Please check that it reads correctly and supply a revised version if necessary.

Q5. Author: Please provide page range for Refs. [4] and [5].

Q6. Author: Please check whether Ref. [6] is okay as set.

Response to the Queries

Q1. We confirm the funding support information is correct.

Q2. Please replace "These are thermoplastics" by "These two materials are thermoplastics".

Q3. Electro Magnetic (EM)

Q4. Delete "In addition," Start the sentence by" The thickness of ...

Q5. For ref [4] 2018 IEEE International Symposium on Antennas and Propagation \& USNC/URSI National Radio Science Meeting, Boston, MA, 2018, pp. 937.

for ref [5] it has been accepted but not published yet (the conference is in September '18) so we do not have the page number yet. Please cite it as "accepted to the 2018 48th European Microwave Conference (EuMC), Madrid, 2018"

Q6. Yes ref [6] is correct. 